\title{
Serum Anti-Mullerian Hormone, Follicle Stimulating Hormone and Antral Follicle Count Measurement as a Prediction for Pregnancy Rates in IVF/ICSI cycle

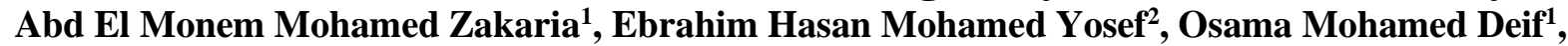 Mohammed Mahmoud Ahmed Khalil ${ }^{1 *}$ \\ ${ }^{1}$ Department of Obstetrics and Gynecology, Faculty of Medicine, Al-Azhar University \\ ${ }^{2}$ Department of Pathology, Faculty of Medicine, Al-Azhar University \\ * Corresponding author: Mohammed Mahmoud Ahmed Khalil, Mobile: (+20) 01067907910, E-mail: drmohamedkhalil86@gmail.com
}

\section{ABSTRACT}

Background: A successful in vitro fertilization treatment depends on the ability of the ovary to respond to gonadotrophin stimulation. This response reflects the ovarian function or 'ovarian reserve'. Poor ovarian response may be associated with reduced number of oocytes and embryos and may lead to cycle cancellation in severe cases. On the other hand, exaggerated response leads to increased risk of ovarian hyper-stimulation syndrome.

Objective: The aim of this study is to assess role of serum AMH, FSH and AFC measurement as a prediction of pregnancy rates in IVF/intracytoplasmic sperm injection (ICSI) cycle.

Patients and Methods: A prospective cross sectional study was conducted at Specialized Air Forced Hospital in the period between January 2018 and December 2018. The study included 100 unexplained infertility patients undergoing IVF/ICSI treatment cycles. They were 82 patients primary infertility and 18 patients secondary infertility.

Results: Type of infertility showed insignificant difference between the 2 groups while menstrual history and gonadotrophins type showed significant difference. Age and duration of infertility were significantly higher in nonpregnant group than pregnant. The mean value of FSH, day 3 E2 levels did not have any significant difference between the 2 group while the mean value of serum E2 the day of HCG injection, serum AMH day 3, AFC were significantly higher in pregnant group than non-pregnant.

Conclusion: No test can predict pregnancy in high accuracy as it is multifactorial however, mean levels of FSH, E2, a day HCG injection and AFC showed significant difference between pregnant and non-pregnant.

Keywords: Anti-Mullerian Hormone, Follicle Stimulating Hormone, Antral Follicle, Pregnancy Rates, IVF/ICSI cycle.

\section{INTRODUCTION}

The reproductive capacity of a woman depends on many factors. Prediction of ovarian reserve has long been the golden key of reproductive endocrinology. Various endocrine follicle stimulating hormone (FSH), inhibin B, estradiol (E2) and ultrasound tests ovarian volume, antral follicle counts (AFC) have been suggested to improve prediction of oocyte yield and pregnancy outcome following assisted reproductive technologies (ART). Currently, most in vitro fertilization (IVF) clinicians determine starting doses of gonadotropin in the first cycle of IVF based principally on the patient's age and basal FSH levels. Ovarian reserve is determined by the size of the ovarian follicle pool and the quality of the produced oocytes. Its declines resulting in a decrease in a woman's reproductive ability with time. Assessing ovarian reserve is a useful but challenging process in the field of IVF. Various hormonal tests have been used, such as basal (day 3) FSH levels, anti-Mullerian hormone levels (AMH), and inhibin B levels. The most commonly used is basal FSH, which is reversely correlated with ovarian reserve. Ultrasonography is also used to assess the quantitative aspect of ovarian reserve ${ }^{(\mathbf{1})}$.

During the last few years, AMH has emerged as one of the most important clinical markers for ovarian reserve in ART. It has a strong correlation with the number of follicles, it is operator independent, can predict reproductive lifespan and it is useful as baseline assessment preceding ovarian stimulation for individualizing the therapeutic strategy. Through paracrine mediation, AMH contributes to control follicle development from a reserve of primordial follicles constituted early in life AMH has been considered an ovarian reserve marker that can be measured independently of the cycle phase with minimal fluctuations in the menstrual cycle ${ }^{(2)}$.

The AFC may reflect the size of the remaining primordial pool in women with proven natural fertility and is highly correlated to the number of oocytes retrieved. Otherwise, AFC can be used in the prediction of ovarian response but not of oocyte/embryo quality or IVF outcome ${ }^{(3)}$.

\section{AIM OF THE WORK}

The aim of this study is to assess role of serum $\mathrm{AMH}, \mathrm{FSH}$ and AFC measurement as a prediction of pregnancy rates in IVF/intracytoplasmic sperm injection (ICSI) cycle.

\section{SUBJECTS AND METHODS}

Study design: A prospective cross sectional study was conducted at Specialized Air Forced Hospital in the period between January 2018 and December 2018. Patients' characteristics: The study included 100 unexplained infertility (UEI) patients undergoing IVF/ICSI. 


\section{Inclusion criteria:}

- Patient less than 40 years old.

- No previous trial of IVF/ICSI.

- $\mathrm{FSH}>9 \mathrm{MIU} / \mathrm{ml}$.

- Normal BMI (19-21).

\section{Exclusion criteria:}

- Patient above 40 years old.

- Past or current hepatic, renal, adrenal or thyroid disorders affecting ovaries or gonadotropin or sex steroid secretion, clearance or excretion.

- Male factor (Azoospermia).

- Patient with morphological abnormalities oocytes which detected by transmission electron microscope imaging.

Ethical approval and written informed consent: An approval of the study was obtained from AlAzhar University Academic and Ethical Committee. Every patient signed an informed written consent for acceptance of the operation.

Before enrolling the patient into assisted reproductive program, a full history was taken from all patients going to systemic clinical examination to assess the general condition and local pelvic physical finding.

\section{Hormonal assessment:}

Blood samples were collected during the early follicular phase of menses preceding the cycle of IVF In all women serum AMH day 3, serum FSH day 3, serum LH day 3, serum E2 day 3 and serum progesterone measured on day of HCG injection may predict IVF outcomes.

AMH concentration was measured in day 2 or 3 of cycle preceding stimulation with an enzymatically amplified two-sided immunoassay \{DSL-10-14400 Active Mullerian Inhibiting substance/AMH enzymelinked immunosorbent assay (ELISA) kits. Diagnostic systems laboratories (DSL), Webster, TX , immulon 2 plates (Dynatech Corp,Chantilly,VA) were coated with monoclonal antibody 10.6 (Dr. R. Cate, Cambridge, MA), raised against recombinant human AMH (rhAMH) by an overnight incubation, at room temperature. Sera were assayed at 1:4, 1:8, 1:16, and 1:32 dilutions in PBS containing 1\% BSA. As second antibody, we used polyclonal antibody L40, consisting of an $\mathrm{IgG}$ fraction isolated by affinity chromatography on protein A-Sepharose from serum of a rabbit immunized with recombinant human $\mathrm{AMH}$ (rhAMH). L40 was added to the wells at $1 \mathrm{ug} / \mathrm{ml}$ in PBS-1\% BSA for 1 hour at room temperature.

Subsequently, an alkaline phosphates-labeled goat anti-rabbit IgG antibody (Jackson Immunoresearch Laboratories, Inc. West Grove, PA) was added and incubated for a further hour before the reaction was visualized with a MRX spectrophotometer (Dynatec Corp.) at $405 \mathrm{~nm}$, using para nitrophenyl phosphate (Sigma Chimie, SaintQuentin-Fallavier, France) as substrate.

\section{Measurement of FSH:}

Serum FSH was measured using ELISA (Gambyt-CR, Diagnostic Products Corporation, Los Angeles, CA).The interassay coefficients of variation FSH was $3.7 \%$. The intra-assay coefficients of variation was $4.7 \%$.

\section{Measurement of E2:}

Estradiol levels were measured by ELISA. Duplicate aliquots $(0.2 \mathrm{ml})$ of plasma were fixed with $0.1 \mathrm{ml}$ of $1.5-\mathrm{mol} / \mathrm{L}$ sodium carbonate solution, $\mathrm{pH}$ 10.5 , and extracted with 10 volumes of diethyl ether. The mixture was frozen, the ether was decanted and evaporated, and the residue was dissolved in phosphate-buffered saline and incubated with tritiumlabeled E2 (Amersham International, Little Chalfont, Buckinghamshire, UK) and sheep anti-E2 antiserum (Bioclin Services, Cardiff, Wales, UK). Bound and free E2 were separated using dextran coated charcoal. The interassay coefficients of variation for two control plasmas were $9.5 \%$ and $6.6 \%$ for E2 concentrations of 174 and $409 \mathrm{pmol} / \mathrm{L}$ respectively.

\section{Measurement of P4:}

Serum progesterone was measured in day of HCG injection using ELISA (Gambyt-CR, Diagnostic products Corporation, Los Angeles, CA). The intraassay and interassay $\mathrm{CV}$ were $9.6 \%$ and $4.9 \%$. All results were taken individually without knowing the others serum progesterone measured in day after HCG administration.

\section{Ultrasound:}

Transvaginal 7.5 MHz (MEDISON) at EIFC IVF center was done early in the cycle to measure the AFC and during induction to follow up follicular growth by the same physician.

Transvaginal ultrasound scans of the ovaries were performed by experienced sonographers who participated in the study.

\section{Controlled ovarian stimulation protocol:}

Standard long GnRH agonist down regulation protocol was used. The stimulation protocol and doses of gonadotropins were not standardized for the study; the treating physician according to age, body mass index, basal FSH level, and AFC and ovarian response made the decision. For the long protocol $0.1 \mathrm{mg}, \mathrm{SC}$, Decapeptyl (Ferring pharmaceuticals, Germany) was started in the midluteal phase. At suppression, ovarian stimulation was initiated with HP- FSH (HP FSH; Fostimon; IBSA, Egypt) from cycle day 2 or 3 and continued until the day of ovulation induction. When at least two follicles had reached a size of $18 \mathrm{~mm}$, HCG (10,000 IU IM, Chorimon 5000 IU, IBSA, Italy) 
has been administrated for final oocyte maturation, followed by OPU 34-36 hours later. Similar luteal support was provided for all patients with intramuscular administration of $100 \mathrm{mg}$ progesterone (Prontogest $100 \mathrm{mg} / 2 \mathrm{ml}$, EIPICO, Egypt) starting on the same day of oocyte retrieval until pregnancy test or until 11 weeks in case pregnancy was achieved.

\section{Transmission electron microscope imaging}

Human oocytes were fixed in $2.5 \%$ glutaraldehyde and $4 \%$ paraformaldehyde (Electron Microscopy Sciences) containing $5 \mathrm{mM} \mathrm{CaCl} 2$ and $0.1 \%$ tannic acid in $0.1 \mathrm{M}$ cacodylate buffer, $\mathrm{pH} 7.4$, for $2 \mathrm{~h}$ at room temperature. They were rinsed twice for $15 \mathrm{~min}$ with the buffer and post-fixed with $1 \%$ osmium tetroxide and $1.5 \%$ potassium ferricyanide in the same buffer for $2 \mathrm{~h}$ at $41 \mathrm{C}$. HOs were then dehydrated in ascending alcohols, treated with propylene oxide and embedded in Araldite (Electron Microscopy Sciences). Ultrathin sections of the samples (80 nm thickness) were cut with a Top Ultra150 ultramicrotome (Pabish) and collected on 300-mesh copper grids. The grids were stained with uranyl acetate and lead citrate and examined at $80 \mathrm{kV}$ using a Jeol Jem 100S transmission electron microscope.

\section{Statistical analysis}

Analysis of data was done by IBM computer using SPSS (Statistical package for social science) version 12 follows:

- Description of quantitative variables as mean , SD and range

- Description of qualitative variables as number and percentages.

- Unpaired t-test was used to compare quantitative variables between two independent groups.

- $P$ value $>0.05$ insignificant.

- P value $<0.05$ significant.

- $\quad$ P value $<0.01$ highly significant.

\section{RESULTS}

The study included measurement of basal serum FSH day 3, serum estradiol both day 3 and on day of $\mathrm{HCG}$ injection and the change between them, basal $\mathrm{AMH}$ and serum progesterone on day of $\mathrm{HCG}$ injection in addition to AFC which were studied for their use in predicting the ovarian reserve.

Table (1): baseline characteristic of included patients

\begin{tabular}{|l|c|c|}
\hline Age (mean \pm SD) & \multicolumn{2}{|c|}{$33.6 \pm 5.3$} \\
\hline Frequency & $\%$ \\
\hline Type of infertility & & \\
\hline Primary & 82 & 82.0 \\
\hline Secondary & & 18.0 \\
\hline Menstrual history & 25 & 25.0 \\
\hline Regular & 75 & 75.0 \\
\hline Irregular & & \\
\hline Gonadotrophins type & 90 & 90.0 \\
\hline HMG & 10 & 10.0 \\
\hline HP.FSH & & \\
\hline Chemical pregnancy rate & 36 & 36.0 \\
\hline +VE (good responders) & 64 & 64.0 \\
\hline -VE (poor responders) & & \\
\hline Clinical pregnancy rate (n=36) & 23 & 63.9 \\
\hline Pulsation & 13 & 36.1 \\
\hline Abortion & & \\
\hline Twins (n=22) & 5 & 22.7 \\
\hline Yes & 17 & 77.3 \\
\hline No & \\
\hline
\end{tabular}


ejhm.journals.ekb.eg

Table (2): Hormones and Outcomes of hormonal stimulation

\begin{tabular}{|l|c|l|c|c|c|c|c|}
\hline & \multicolumn{3}{c}{ Range } & \multicolumn{3}{c|}{ Mean \pm SD } & Median \\
\hline FSH (Basal) & 1.3 & - & 77 & 8.2 & \pm & 8.7 & 6.6 \\
\hline LH (Basal) & 2.1 & - & 62 & 10.9 & \pm & 10 & 6.9 \\
\hline E2 (Basal) & 7.4 & - & 80 & 34.1 & \pm & 12.9 & 32.8 \\
\hline AMH (Basal) & 0.06 & - & 10.9 & 1.7 & \pm & 1.9 & 0.9 \\
\hline AFC & 12 & - & 18 & 15.2 & \pm & 1.9 & 16 \\
\hline No. Stimulation days & 12 & - & 15 & 12.8 & \pm & 0.8 & 13 \\
\hline S. progesterone on hCG day & 0.04 & - & 1.4 & 0.6 & \pm & 0.4 & 0.5 \\
\hline S. LH on hCG day & 0.9 & - & 4.2 & 2.3 & \pm & 0.8 & 2.5 \\
\hline S. E2 on hCG day & 1100 & - & 2450 & 2252 & \pm & 1750 & 22582 \\
\hline NO. MII Oocyte & 3 & - & 24 & 7.6 & \pm & 5.0 & 6 \\
\hline NO. Transferred Embryos & 1 & - & 4 & 2.5 & \pm & 1.4 & 2 \\
\hline No. cryopreserved embryos & 0 & - & 10 & 0.6 & \pm & 1.9 & 2 \\
\hline
\end{tabular}

Table (3): Comparison between pregnant and non-pregnant:

\begin{tabular}{|c|c|c|c|c|c|c|c|}
\hline & \multicolumn{3}{|c|}{ Good responder } & \multicolumn{3}{c|}{ Poor responder } & P value \\
& Mean & \multicolumn{2}{|c|}{ Mean } & \multicolumn{2}{c|}{ SD } & \\
\hline Age (years) & 28.0 & \pm & 5.4 & 33.9 & \pm & 5.0 & 0.001 \\
\hline Infertility Duration (years) & 3.0 & \pm & 1.8 & 5.1 & \pm & 1.9 & 0.006 \\
\hline FSH $I U / m l$ (Basal) & 8.3 & \pm & 2.4 & 7.9 & \pm & 1.9 & 0.6 \\
\hline E2 $P g / m l$ (Basal) & 32.0 & \pm & 3.1 & 35.7 & \pm & 2.6 & 0.4 \\
\hline AMH $\mathrm{ng} / \mathrm{ml}$ (Basal) & 3.9 & \pm & 0.5 & 0.7 & \pm & 0.08 & 0.001 \\
\hline AFC ( $\mathrm{mm}$ ) & 17.4 & \pm & 1.4 & 12.0 & \pm & 1.4 & 0.001 \\
\hline Stimulation Duration (days) & 13.7 & \pm & 1.0 & 11.2 & \pm & 0.8 & 0.001 \\
\hline Progesterone & 0.36 & \pm & 0.029 & 0.8 & \pm & 0.35 & 0.002 \\
\hline FSH IU/ml & 2.9 & \pm & 0.9 & 3.2 & \pm & 0.8 & 0.05 \\
\hline E2 $\mathrm{pg} / \mathrm{ml}$ & 2570 & \pm & 12.0 & 876.2 & \pm & 14.2 & 0.001 \\
\hline
\end{tabular}

Table (4): Correlation between different hormones:

\begin{tabular}{|c|c|c|c|c|c|c|c|c|c|}
\hline & & $\begin{array}{l}\text { FSH } \\
\text { Basal }\end{array}$ & $\begin{array}{c}\text { LH } \\
\text { Basal }\end{array}$ & $\begin{array}{c}\text { E2 } \\
\text { Basal }\end{array}$ & $\begin{array}{l}\text { AMH } \\
\text { Basal }\end{array}$ & $\mathrm{AFC}$ & Progesterone & FSH & LH \\
\hline \multirow[t]{2}{*}{ LH Basal } & $\mathrm{r}$ & 0.51 & & & & & & & \\
\hline & $\mathrm{p}$ & 0.001 & & & & & & & \\
\hline \multirow[t]{2}{*}{ E2Basal } & $\mathrm{r}$ & -0.050 & -0.025 & & & & & & \\
\hline & $\mathrm{p}$ & 0.7 & 0.8 & & & & & & \\
\hline \multirow[t]{2}{*}{ AMH Basal } & $\mathrm{r}$ & 0.041 & -0.150 & 0.062 & & & & & \\
\hline & $\mathrm{p}$ & 0.7 & 0.100 & 0.45 & & & & & \\
\hline \multirow[t]{2}{*}{$\mathrm{AFC}$} & $\mathrm{r}$ & 0.07 & 0.01 & 0.07 & 0.35 & & & & \\
\hline & $p$ & 0.4 & 0.8 & 0.4 & 0.001 & & & & \\
\hline \multirow[t]{2}{*}{ Progesterone } & $\mathrm{r}$ & 0.05 & 0.3 & 0.02 & -0.19 & -0.2 & & & \\
\hline & $p$ & 0.5 & 0.03 & 0.9 & 0.1 & 0.04 & & & \\
\hline \multirow[t]{2}{*}{ FSH } & $\mathrm{r}$ & 0.38 & 0.35 & 0.1 & -0.2 & -0.15 & 0.17 & & \\
\hline & $p$ & $<0.001$ & $<0.001$ & 0.3 & 0.010 & 0.19 & 0.08 & & \\
\hline \multirow[t]{2}{*}{$\mathrm{LH}$} & $\mathrm{r}$ & 0.3 & 0.3 & 0.15 & -0.09 & -0.2 & 0.19 & 0.7 & \\
\hline & $p$ & 0.004 & 0.004 & 0.165 & 0.385 & 0.055 & 0.242 & $<0.001$ & \\
\hline \multirow[t]{2}{*}{ E2 } & $\mathrm{r}$ & -0.02 & 0.02 & -0.04 & 0.04 & -0.07 & 0.2 & 0.15 & 0.15 \\
\hline & p & 0.8 & 0.8 & 0.7 & 0.6 & 0.5 & 0.05 & 0.1 & 0.1 \\
\hline
\end{tabular}


Table (5): Correlation of hormones between oocytes and Pregnancy:

\begin{tabular}{|c|c|c|c|}
\hline & & NO of Oocyte MII & Pregnancy \\
\hline \multirow[t]{2}{*}{ Basal FSH( Iu/ml) } & $\mathrm{r}$ & -0.04 & 0.07 \\
\hline & $\mathrm{P}$ & 0.7 & 0.6 \\
\hline \multirow[t]{2}{*}{ Basal LH( Iu/ml) } & $\mathrm{r}$ & -0.15 & -0.05 \\
\hline & $\mathrm{P}$ & 0.2 & 0.7 \\
\hline \multirow{2}{*}{ Basal E2 $(p g / m l)$} & $\mathrm{r}$ & 0.1 & 0.05 \\
\hline & $\mathrm{P}$ & 0.3 & 0.6 \\
\hline \multirow{2}{*}{ Basal AMH( $n g / m l)$} & $\mathrm{r}$ & 0.4 & 0.6 \\
\hline & $\mathrm{P}$ & 0.001 & 0.001 \\
\hline \multirow[t]{2}{*}{$\mathrm{AFC}(m m)$} & $\mathrm{r}$ & 0.4 & 0.5 \\
\hline & $\mathrm{P}$ & 0.001 & 0.001 \\
\hline \multirow[t]{2}{*}{ Stimulation Duration (days) } & $\mathrm{r}$ & -0.1 & 0.2 \\
\hline & $\mathrm{P}$ & 0.1 & 0.04 \\
\hline \multirow[t]{2}{*}{ Progesterone $(n g / m l)$} & $\mathrm{r}$ & -0.2 & -0.3 \\
\hline & $\mathrm{P}$ & 0.03 & 0.002 \\
\hline \multirow[t]{2}{*}{$\mathrm{FSH}(\mathrm{Iu} / \mathrm{ml})$} & $\mathrm{r}$ & -0.1 & -0.2 \\
\hline & $\mathrm{P}$ & 0.29 & 0.05 \\
\hline \multirow[t]{2}{*}{$\mathrm{LH}(\mathrm{Iu} / \mathrm{ml})$} & $\mathrm{r}$ & -0.1 & -0.09 \\
\hline & $\mathrm{P}$ & 0.1 & 0.3 \\
\hline \multirow[t]{2}{*}{$\mathrm{E} 2(p g / m l)$} & $\mathrm{r}$ & -0.1 & -0.1 \\
\hline & $\mathrm{P}$ & 0.2 & 0.2 \\
\hline \multirow[t]{2}{*}{ NO of MII Oocytes } & $\mathrm{r}$ & & 0.3 \\
\hline & $\mathrm{P}$ & & 0.001 \\
\hline
\end{tabular}

Table (6): Ability of AMH to predict good responders:

\begin{tabular}{|l|l|l|l|}
\hline \multicolumn{2}{|c|}{ A: AMH area under the curve (AUC) and p value } \\
\hline Test variable & AUC & $95 \%$ CI for AUC & P value \\
\hline AMH & 0.800 & $0.745-0.876$ & 0.001 \\
\hline
\end{tabular}

B: $P P V=$ positive predictive value, $\mathrm{NPV}=$ negative predictive value.

\begin{tabular}{|l|l|l|l|l|l|}
\hline Cut-off point & Sensitivity & Specificity & PPV & NPV & Accuracy \\
\hline$\geq 1.3$ & $85.8 \%$ & $92.0 \%$ & $83.8 \%$ & $93.0 \%$ & $89.0 \%$ \\
\hline
\end{tabular}




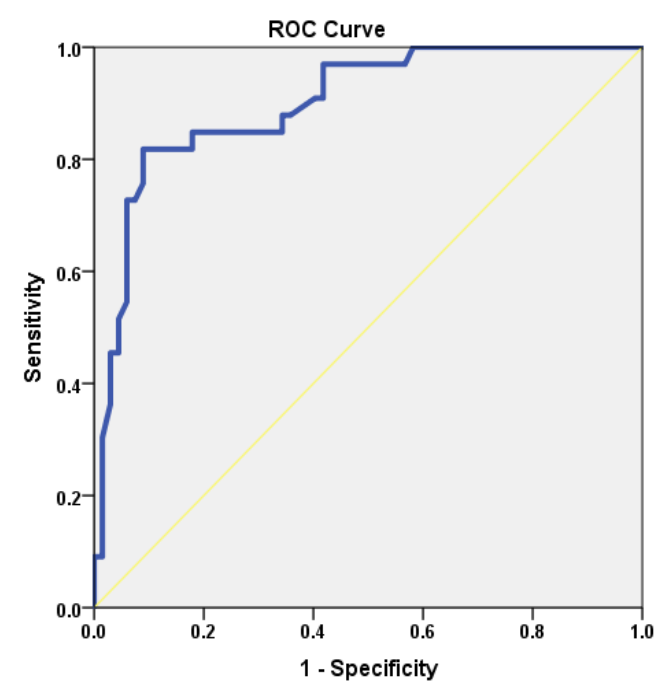

Fig. (1): Ability of AMH to predict good responders.

Table (8): Ability of AFC to predict good responders:

A: AUC =Area under the curve, $\mathrm{CI}=$ confidence interval.

\begin{tabular}{|l|l|l|l|}
\hline Test variable & AUC & 95\% CI for AUC & P value \\
\hline
\end{tabular}

\begin{tabular}{|c|c|c|c|}
\hline $\mathrm{AF}$ count & 0.8 & $0.635-0.855$ & $<0.001$ \\
\hline
\end{tabular}

\section{B: $P P V=$ positive predictive value, $\mathrm{NPV}=$ negative predictive value.}

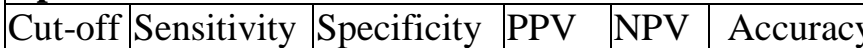

\begin{tabular}{|l|l|l|l|l|l|}
$\begin{array}{l}\text { Cut-off } \\
\text { point }\end{array}$ & Sensitivity & Specificity & PPV & NPV & Accuracy \\
$\geq 10$ & $100.0 \%$ & $39.3 \%$ & $44.9 \%$ & 100.0 & $60.0 \%$ \\
$\%$ & &
\end{tabular}

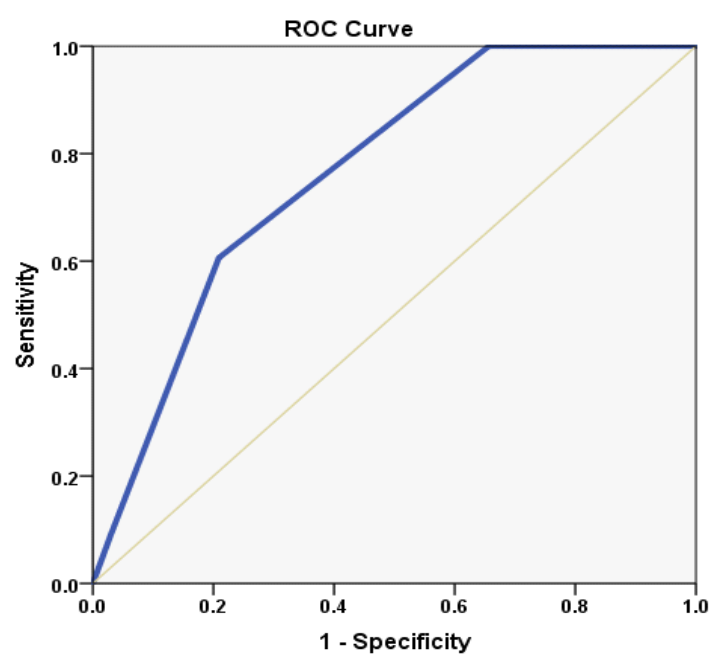

Fig. (2): Ability of AFC to predict good responders.

\section{DISCUSSION}

AMH in the prediction of pregnancy has been investigated in various studies which showed inconsistent results. Some studies suggest that serum AMH level is associated with pregnancy rates; whereas others suggested that serum AMH levels are not associated with pregnancy outcomes. Other markers such as AFC and inhibin B, which were thought to predict pregnancy, were also evaluated in many studies ${ }^{(4-7)}$. In our study we detected that day 3 serum AMH, AFC, and inhibin B measurements in normal responder women were not associated with pregnancy rates.

The present study type of infertility did not have any statistically significant difference between the 2 groups while menstrual history and gonadotrophins type had a highly significant difference. Age and duration of infertility were significantly higher in nonpregnant group than pregnant with. The mean value of FSH, day 3 E2 levels did not have any statistically difference between 2 group. The mean value of serum E2 the day of HCG injection, serum AMH day 3, AFC were significantly higher in pregnant group than nonpregnant. Mean value of serum progesterone day HCG injection was significantly higher in nonpregnant group than pregnant. Inverse correlation was observed between oocyte and basal FSH, LH, weak correlation with E2. In pregnancy correlation between basal FSH, LH, and E2 were poor. Serum AMH, AFC correlated with oocyte and pregnancy while serum progesterone inversely correlated with oocyte and pregnancy. Stimulation days and serum (FSH, LH and E2) after stimulation inversely correlated with oocyte while correlated with pregnancy. Oocyte correlated strongly with pregnancy.

In a study by Yarali $\boldsymbol{e t}$ al. ${ }^{(4)}$ they evaluated ICSI outcome in women over 39 years and wanted to determine when to discourage such couples to undergo IVF using their own oocytes. Four hundred ninety-five consecutive women $(n=495)$ over the age of 39 were evaluated year-by-year age increment to discriminate the independent prognostic factors for the achievement of pregnancy. Clinical pregnancy rates per embryo transfer decreased from $26 \%$ to $13 \%$ from age 40 to 44 . The female age seems to be the only variable in order to predict an ongoing pregnancy. The miscarriage rate increased with advancing female age. It was $33 \%$ at age 40 but increased to $100 \%$ by age 45 . They concluded that performing controlled ovarian hyperstimulation $(\mathrm{COH})$ and embryological data is not discouraging among women over 39 years in ICSI cycle. However, increased miscarriages as well as decreased implantation rate are mainly responsible for the poor performance of patients with advanced female age. Irrespective of the ovarian reserve testing, ICSI may be refused at age 45 and thereafter ${ }^{(4)}$.

Poor ovarian responders have less chance of getting pregnant; this was detected through good responder patients (36 pregnant women) had $80.0 \%$ primary infertility while $20.0 \%$ had secondary infertility, $57.1 \%$ were in regular cycle while $42.9 \%$ in irregular cycle. In poor responder's patients (64 
non-pregnant women) $83.0 \%$ had primary infertility while $17.0 \%$ had secondary infertility, $12.3 \%$ were in regular cycle while $87.7 \%$ in irregular cycle. Hammadeh et al. ${ }^{(5)}$ reported that secondary infertility was a good prognostic factor for IVF outcomes in terms of biochemical pregnancy.

Hazout et al. ${ }^{(6)}$; evaluated 109 women ( $<42$ years old) and demonstrated that day 3 serum AMH level and IVF outcome were strongly associated, and higher AMH concentrations were associated with a higher clinical pregnancy rate; moreover, they showed that AMH might offer greater prognostic value than other currently available serum markers of ART outcome.

Wu et al. ${ }^{(7)}$; detected that day $3 \mathrm{AMH}$ and AFC were significantly higher in pregnant women compared to non-pregnant women (total of 60 infertile women). Multiple regression analysis for prediction of pregnancy showed day $3 \mathrm{AMH}$ to be a good predictor of clinical pregnancy. The latter three studies had a common limitation: a small number of cases.

Majumder et al. ${ }^{(8)}$; prospectively evaluated 162 infertile women and observed that both day 3 AMH and AFC had highly significant correlations with the number of oocytes retrieved and the number of oocytes fertilized. AMH was better than AFC in terms of predicting live birth, but both markers were more valuable in predicting the absence rather than the occurrence of live birth.

Several authors suggested that measurement of follicular fluid AMH and outpatient unit day serum AMH could predict fertilization and clinical pregnancy rates. However, prediction of pregnancy on day of OPU is too little too late since the IVF cycle has already been completed ${ }^{(9)}$.

Broer et al. ${ }^{(10)}$; performed a meta-analysis of 13 trials on $\mathrm{AMH}$ and 17 trials on AFC. They detected that sensitivities and specificities of $\mathrm{AMH}$ for the prediction of poor ovarian response varied between $40 \%$ and $91 \%$ and between $64 \%$ and $100 \%$, respectively. Moreover, the receiver operating characteristic (ROC) curves do not suggest a clearly better predictive ability for AMH than for AFC, and the difference was not statistically significant $(P=0.73)$. The authors concluded that AMH has at least the same level of accuracy and clinical value for the prediction of poor response and non-pregnancy as AFC. All of the above studies were agreed with results of this studies.

There are few studies which suggest that serum AMH is not associated with ongoing pregnancy rates. Penarrubia et al. (11); compared the data of 20 cancelled cycles and 60 controls and showed that basal and day $5 \mathrm{AMH}$ serum concentrations were significantly lower in the cancelled than in the control group; and the capacity of day $5 \mathrm{AMH}$ in predicting the likelihood of cancellation in an ART program was significantly higher than that for basal $\mathrm{AMH}$ measurement. However, in this study, AMH was not found to be beneficial in the prediction of pregnancy.

Deffieux and Antoine (12); suggested that day 3 AMH levels predict the number of oocytes retrieved, but the AMH level cannot predict the likelihood of pregnancy.

Means of AMH differ in various studies. The most striking study on means of $\mathrm{AMH}$ in general population is the study of Tremellen and Kolo ${ }^{(13)}$. They evaluated a total of 1032 women aged between 18 and 43 years and found that the mean serum AMH level is relatively stable at approximately $30 \mathrm{pmol} / \mathrm{l}$ $(4.1 \mathrm{ng} / \mathrm{ml})$ (1 ng AMH is $7.143 \mathrm{pmol})$ in the under 30-year-old range; however, from 30 years of age onwards the serum AMH levels decline rapidly, halving in concentration to an average of only 14 $\mathrm{pmol} / \mathrm{L}(1.95 \mathrm{ng} / \mathrm{ml})$ in the 35 - to 39-year-old age group $^{(13)}$.

In the present study, we also evaluated the clinical pregnancy rates according to the quartiles of $\mathrm{AMH}$. We observed that clinical pregnancy rates tended to increase with increasing quartiles of serum $\mathrm{AMH}$, but differences between the pregnancy rates of $25 \%, 50 \%$ and $75 \% \mathrm{AMH}, \mathrm{FSH}$, and AFC groups were statistically non -significant.

The pregnancy rate was $21 \%$ in patients whose serum AMH level was lower than $1.81 \mathrm{ng} / \mathrm{ml}$ and $29.2 \%$ in patients whose serum AMH levels were higher than $4.92 \mathrm{ng} / \mathrm{ml}$. The lowest level of serum $\mathrm{AMH}$ was $0.45 \mathrm{ng} / \mathrm{ml}$ in the pregnant group. Considering our results, we may suggest that $\mathrm{AMH}<$ $1 \mathrm{ng} / \mathrm{ml}$ does not definitely predict conception failure.

In a study by Wang et al. (14) who retrospectively investigated the relationship between IVF clinical pregnancy rates per initiated cycle and serum AMH tertile stratified by age in 1558 women in all age groups and detected that age influenced the AMH and clinical pregnancy rate relationship.

They found that for women aged $>42$ years with $\mathrm{AMH}<0.29 \mathrm{ng} / \mathrm{ml}$, the clinical pregnancy rate was significantly lower than those of the middle and higher quartiles. However, our results may only be appropriate for women younger than 40 years, since older women were excluded.

Taking $\mathrm{P}$ value $<0.001$, AFC was considered highly significant for poor response. In ROC curve analysis area under the curve was $>75 \%$; the value of testing AFC in predicting response was high. With optimum cutoff point $>10$, the highest sensitivity and specificity that could be obtained were $100 \%$ and $39.3 \%$ respectively. The accuracy was $60 \%$. Basal FSH in our study was not significant for both poor and high response. Basal serum estradiol level day 3 could not predict response. Serum estradiol day $\mathrm{HCG}$ injection and the difference from day hCG injection to day 3 reflects the number and health of oocyte and could be used to get reassuring results about response 
and pregnancy. In our study we did not categorized these levels, however, literature proved the value of categorization. Measuring serum progesterone in day of HCG injection was highly significant.

AMH measurement proved to be effective in predicting response of patients. However, we advise against routing measurement of $\mathrm{AMH}$ because it is expensive and AFC is more accurate. In ROC curve analysis area under the curve was $>75 \%$ i.e. the value of testing $\mathrm{AMH}$ in predicting response is low. At cutoff value of $>1.3 \mathrm{ng} / \mathrm{ml}$ the sensitivity was $85.8 \%$, specificity was $92 \%$ and the accuracy was $89.0 \%$.

\section{CONCLUSIONS}

- Testing of ovarian reserve prior to IVF program is a worthy procedures as it saves unnecessary procedures, canceled cycles, wasted resources, and emotional stress to the patient in case of low estimate and on the other hand can help adjusting the doses of ovarian stimulation to obtain the appropriate response.

- Age is one the most established markers of ovarian reserve and in our study the age of 38 years seems to be a cutoff point after which results of IVF is highly impaired.

- Longer duration of infertility and primary infertility CAM is a sign of underlying poor ovarian reserve. Induction in poor ovarian reserve patients will need more doses of HMG.

- Among variable ovarian reserve test taken in our study we found that transvaginal ultrasonography done in the early follicular phase (on day 2-5 of the cycle) prior to induction of ovulation can be considered an easy, helpful non-invasive and cheap method in prediction of ovarian response to ovulation induction by gonadotropins in IVF patients.

- No test can predict pregnancy in high accuracy as it is multifactorial however, mean levels of FSH, E2, a day HCG injection and AFC showed statistical significant difference between pregnant and non-pregnant.

\section{REFERENCES}

1. Kotanidis L, Nikolettos K, Petousis S et al. (2016): The use of serum anti-Mullerian hormone (AMH) levels and antral follicle count (AFC) to predict the number of oocytes collected and availability of embryos for cryopreservation in IVF. Journal of Endocrinological Investigation, 39(12): 1459-1464.

2. Melado L, Lawrenz B, Bungum $L$ et al. (2018): Antimüllerian hormone variability during ovarian stimulation for IVF. Journal of Reproductive Biology and Endocrinology, 2(1):33-34.
3. Hassan A, Kotb M, AwadAllah A et al. (2017): Follicular output rate can predict clinical pregnancy in women with unexplained infertility undergoing IVF/ICSI: a prospective cohort study. Reproductive Biomedicine Online, 34(6): 598-604.

4. Yarali H, Bozdag G, Polat M et al. (2010): Intracytoplasmic sperm injection outcome of women over 39: an analysis of 668 cycles. Archives of Gynecology and Obstetrics, 281(2): 349-55.

5. Hammadeh ME, Fischer-Hammadeh C, Ali KR (2011): Assisted hatching in assisted reproduction: a state of the art. Journal of Assisted Reproduction and Genetics, 28(2): 119-128.

6. Hazout A, Bouchard P, Seifer DB et al. (2004): Serum antimüllerian hormone/müllerian-inhibiting substance appears to be a more discriminatory marker of assisted reproductive technology outcome than follicle-stimulating hormone, inhibin B, or estradiol. Fertility and Sterility, 82(5): 1323-1329.

7. Wu CH, Chen YC, Wu HH et al. (2009): Serum antiMüllerian hormone predicts ovarian response and cycle outcome in IVF patients. Journal of Assisted Reproduction and Genetics, 26(7): 383-389.

8. Majumder K, Gelbaya TA, Laing I et al. (2010): The use of anti-Müllerian hormone and antral follicle count to predict the potential of oocytes and embryos. European Journal of Obstetrics \& Gynecology and Reproductive Biology, 150(2): 166-170.

9. Fanchin R, Schonäuer LM, Righini C et al. (2003): Serum anti- Müllerian hormone is more strongly related to ovarian follicular status than serum inhibin B, estradiol, FSH and LH on day 3. Human Reproduction, 18(2): 323327.

10. Broer SL, Mol BWJ, Hendriks D et al. (2009): The role of antimullerian hormone in prediction of outcome after IVF: comparison with the antral follicle count. Fertility and Sterility, 91(3): 705-714.

11. Penarrubia J, Fábregues F, Manau D et al. (2005): Basal and stimulation day 5 anti-Müllerian hormone serum concentrations as predictors of ovarian response and pregnancy in assisted reproductive technology cycles stimulated with gonadotropin-releasing hormone agonistgonadotropin treatment. Human Reproduction, 20(4): 915-922.

12. Deffieux X, Antoine J (2003): Inhibins, activins and antiMüllerian hormone: structure, signalling pathways, roles and predictive value in reproductive medicine. Gynecologie, Obstetrique \& Fertilite, 31(11): 900-911.

13. Tremellen K, Kolo M (2010): Serum anti- Mullerian hormone is a useful measure of quantitative ovarian reserve but does not predict the chances of live- birth pregnancy. Australian and New Zealand Journal of Obstetrics and Gynaecology, 50(6): 568-572.

Wang JG, Douglas NC, Nakhuda GS et al. (2010): The association between anti-Müllerian hormone and IVF pregnancy outcomes is influenced by age. Reproductive Biomedicine Online, 21(6): 757-761. 